IDDF2018-ABS-0051 LONG-TERM OUTCOMES OF LIMITED ENDOSCOPIC SPHINCTEROTOMY PLUS LARGE-BALLOON DILATION VERSUS ENDOSCOPIC PAPILLARY LARGE-BALLOON DILATION ALONE FOR REMOVAL OF BILE DUCT STONES

Tao Li*. Digestive Endoscopy Center, Department of Gastroenterology, Ruijin Hospital, Affiliated to Shanghai Jiao Tong University School of Medicine, China

10.1136/gutjnl-2018-IDDFabstracts.197

Background Limited endoscopic sphincterotomy with large balloon dilation (ES-LBD) and endoscopic papillary large-balloon dilation (EPLBD) alone have been proven safe and effective for removal of common bile duct (CBD) stones. However, few reports exist regarding the long-term outcomes of these techniques. The aim of this study was to assess long-term outcomes of ES-LBD compared with EPLBD alone for retrieval of CBD stones.

Methods Patients with EPLBD alone or ES-LBD referred for CBD stones removal between June 2008 and August 2015 in our centre were retrospectively reviewed. The main outcomes of complete stone clearance, ERCP-related adverse events, late biliary complications during long-term follow-up were analysed.

Results Basic patient characteristics were not significantly different between the groups that underwent EPLBD alone $(n=164)$ and ES-LBD $(n=52)$. EPLBD alone compared with ES-LBD resulted in similar outcomes in terms of overall successful stone removal $(99.4 \%$ vs $100 \%, \mathrm{p}=0.76)$ and ERCPrelated adverse events $(7.9 \%$ vs $5.8 \%, \mathrm{p}=0.77)$. The mean duration of the follow-up was 70.5 months and 65.1 months who underwent EPLBD alone and ES-LBD, respectively $(p=0.17)$. A significantly higher incidence of late biliary complications was observed in the ES-LBD group than EPLBD alone group (12 [23.1\%] vs 18 [11.0\%]; p=0.04). Multivariate analysis showed that endoscopic sphincterotomy (EST) (OR, 2.407; 95\% CI, 1.054-5.501; $\mathrm{p}=0.037)$ and mechanical lithotripsy (ML) (OR, 2.981; 95\% CI, 1.272-6.987; p=0.012) were independent risk factors for late biliary complications. Conclusions During long-term follow-up, patients who underwent ES-LBD had significantly more late biliary complications than those after EP-LBD alone. EST and ML, special attention should be paid to the possible occurrence of late biliary complications.

\section{IDDF2018-ABS-0054 TIPSS IN DIFFICULT-TO-TREAT PLEURAL EFFUSION IN CIRRHOSIS. DOES HEPATIC HEMODYNAMICS RESPONSE HAVE A ROLE?}

Ankur Jindal* ${ }^{*}$ Amar Mukund. Institute of Liver and Biliary Sciences, New Delhi, India

\subsection{6/gutjn|-2018-IDDFabstracts.198}

Background Pleural effusions complicate advanced liver disease in $5 \%$ patients. Early identification of cause and related complications is imperative for appropriate management and survival. Unlike refractory ascites, data is limited on safety and efficacy of TIPS in cirrhotics with refractory refilling pleural effusion.
Methods We analysed a consecutive cohort of hospitalised cirrhotics having pleural effusion(PE) at admission. Baseline HVPG and PE tap were done for aetiology and any infection. We determined rate and predictors of $\mathrm{PE}$ resolution with standard medical treatment(SMT) need for intercostal drainage (ICD), and efficacy and safety of TIPS in HH.

Results Of 1149 admissions involving 762 cirrhotics (mean CTP-10.6) with PE, 967 (84.2\%) had hepatic hydrothorax (HH),181 (15.8\%) tubercular PE (TBPE) and despite comparable HVPG, CTP and MELD scores at baseline, patients with $\mathrm{HH}$ compared to TBPE developed more complications (HE$41.6 \%$ vs $30.2 \%$, AKI- $48.6 \%$ vs $37 \%$ and septic shock$14.1 \%$ vs $8.3 \%$; all $\mathrm{p}<0.01) .475(49.2 \%) \mathrm{HH}$ were symptomatic (dyspnea-30.1\%, cough-24\%, chest pain-16.9\%) at admission and PE tap revealed SBE in $22.9 \%$. Presence of coexisting SBP (52.5\%;OR: 5.2) and ICD placement $24.2 \%$; OR: 3.1) were independent predictors for SBE. Baseline HVPG (16.6 \pm 4.4 vs. 16.4? 5.1; P-0.6) and MELD scores were comparable in SBE and no SBE. 43\% patients of $\mathrm{HH}$ responded to SMT alone, $13.8 \%$ required ICD. 41 patients underwent TIPS [based on lower CTP- 9.9 \pm 1.6 and MELD-

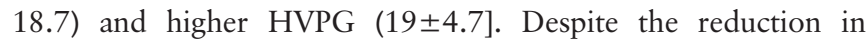
pressure gradient (mean PV-RAP) from 23.1 to $7.2 \mathrm{mmHg}$ ), only $20(48.2 \%)$ had complete resolution of $\mathrm{HH}$, with similar mortality. Main post-TIPScomplications were encephalopathy (8 patients, 6 resolved) and ischaemic hepatitis (4 patients, 2 resolved). 321 (35.9\%) HH patients had in-hospital mortality and independent predictors were MELD >25, SBE nonresponse to SMT and septic shock.

Conclusions Only one-half of $\mathrm{HH}$ resolve with standard medical therapy and need for any intervention including TIPS generally heralds poor outcome. Role of hepatic hemodynamics response in predicting complications and resolution to $\mathrm{HH}$ is limited. Early referral for liver transplantation is imperative.

\section{IDDF2018-ABS-0064 COMPARISON OF CHILD-PUGH AND MODEL FOR END STAGE LIVER DISEASE SCORES AS PREDICTOR OF SPONTANEOUS BACTERIAL PERITONITIS}

${ }^{1}$ Muhammad Manko*, ${ }^{2}$ Gadzama Bala Galadima, ${ }^{3}$ Adamu Alhaji Samaila, ${ }^{3}$ Muhammad Musa Borodo. 'Department of Medicine Ahmadu Bello University Zaria, Nigeria; ${ }^{2}$ Department of Medical Microbiology University of Maiduguri, Maiduguri, Nigeria; ${ }^{3}$ Department of Medicine Bayero University Kano, Nigeria

\subsection{6/gutjnl-2018-IDDFabstracts.199}

Background Spontaneous bacterial peritonitis (SBP) is the presence of ascitic fluid infection in the absence of any surgically treatable source. SBP is a common complication of liver cirrhosis.

Aim To compare Child-Pugh (CP) and Model for End Stage Liver Disease (MELD) scores as a predictor of SBP.

Subjects Adult liver cirrhosis patients with ascitis who had no antibiotic usage in the previous one month seen at the gastroenterology unit of Aminu Kano Teaching Hospital, Kano.

Methods The study is a cross-sectional study of 170 consecutive patients seen between April 2015 to October 2016. Detailed history taking and physical examination were carried out on all patients. Blood samples were taken for liver function test including serum bilirubin, international normalised 